\title{
The responsible use of antibiotics for an elevated PSA level
}

\author{
Peter T Scardino
}

PSA levels vary spontaneously, rising and falling an average of $15 \%$ from week to week. A rise of $<20-46 \%$ from one year to the next is more likely to be the result of biological variation than cancer (Ornstein DK et al. [1997] $J$ Urol 157: 2197-2182). Changes in PSA levels are not surprising, since the level in prostatic fluid is nearly a million-fold higher than in serum; leakage from prostatic acini to surrounding capillaries can substantially affect serum levels. Consequently, the widespread practice of prescribing antibiotics for men with a newly elevated PSA level, on the presumption that the patient has infectious prostatitis, seems irrational.

About $10-15 \%$ of men tested will have a PSA level $>4 \mathrm{ng} / \mathrm{ml}$ in any given round of screening. Over multiple tests $21-37 \%$ of men without cancer will have at least one abnormal PSA level, depending on the definition used, however, the level will return to normal at the next test in 26$37 \%$, and will become normal with subsequent testing in 40-55\%. Once normalized, 65-83\% of men have normal PSA levels for several years with no therapy (Eastham JA et al. [2003] JAMA 289: 2695-2700).

Despite the lack of evidence, antibiotics are often prescribed for men with an elevated PSA on the presumption that the elevation is caused by infection (Kaygisiz O et al. [2006] Prostate Cancer Prostatic Dis 9: 235-238). While antibiotics may influence the course of bacterial prostatitis, $90 \%$ of symptomatic prostatitis and almost all cases of asymptomatic prostatitis (NIH Category 4) are not caused by a bacteria (Habermacher GM et al. [2006] Annu Rev Med 57: 195-206). While prostatic inflammation has been associated with increased PSA levels, antibiotics have no effect on nonbacterial prostatitis. In several studies of men with elevated PSA levels, 32-42\% had evidence of Category 4 prostatitis. The PSA level fell to normal in 43$46 \%$ treated with antibiotics and remained so
Despite

the lack of

evidence, antibiotics

are often

prescribed for

men with an elevated PSA

PT Scardino is Editorin-Chief of Nature Clinical Practice Urology.

\section{Competing interests \\ The author declared he has no competing interests.}

www.nature.com/clinicalpractice doi:10.1038/ncpuro0702 in most men for 1-2 years (Bozeman CB et al. [2002] J Urol 167: 1723-1726).

Did the antibiotics affect PSA levels in these men? Almost certainly not; the changes in PSA with antibiotics were similar to the random variations in healthy men. Also, there were no significant differences in bacterial cultures before or after antibiotics between PSA responders and nonresponders (Potts JM [2000] J Urol 164: 1550-1553). None of these studies included a control group, and there have been no randomized trials to show that antibiotics are more likely to lower PSA levels than a placebo.

PSA often falls with the next test whether or not antibiotics have been used, so why is there a widespread belief that antibiotics given to asymptomatic men will lower PSA levels? If PSA levels do not fall, the probability of finding cancer is, in fact, higher than if levels decrease (of course, cancer is more likely in men with a sustained PSA elevation than those with a randomly variable PSA that is temporarily elevated).

What harm is there in taking a course of antibiotics if it makes the patient feel that something is being done? Besides the expense, the risk of toxicity and the propagation of unscientific medical practice, the widespread use of broad spectrum antibiotics promotes the development of resistant organisms and exposes the patient to an increased risk of sepsis from a resistant strain, if undergoing prostate biopsy.

In summary, when an asymptomatic man presents with a modestly elevated PSA level $(<10 \mathrm{ng} / \mathrm{ml})$ and a normal digital rectal examination, the most appropriate recommendation is to reassure the patient, repeat the PSA level once or twice and, if the levels remain elevated, to perform a biopsy. There is no evidence that antibiotics alter PSA levels except in the presence of bacterial prostatitis, which is an uncommon condition. 